\title{
Population-level transcriptome sequencing of nonmodel organisms Erynnis propertius and Papilio zelicaon
}

\author{
Shawn T O'Neil1', Jason DK Dzurisin², Rory D Carmichael1, Neil F Lobo², Scott J Emrich¹ and Jessica J Hellmann²
}

\begin{abstract}
Background: Several recent studies have demonstrated the use of Roche 454 sequencing technology for de novo transcriptome analysis. Low error rates and high coverage also allow for effective SNP discovery and genetic diversity estimates. However, genetically diverse datasets, such as those sourced from natural populations, pose challenges for assembly programs and subsequent analysis. Further, estimating the effectiveness of transcript discovery using Roche 454 transcriptome data is still a difficult task.

Results: Using the Roche 454 FLX Titanium platform, we sequenced and assembled larval transcriptomes for two butterfly species: the Propertius duskywing, Erynnis propertius (Lepidoptera: Hesperiidae) and the Anise swallowtail, Papilio zelicaon (Lepidoptera: Papilionidae). The Expressed Sequence Tags (ESTs) generated represent a diverse sample drawn from multiple populations, developmental stages, and stress treatments.

Despite this diversity, $>95 \%$ of the ESTs assembled into long (> 714 bp on average) and highly covered (> 9.6 $x$ on average) contigs. To estimate the effectiveness of transcript discovery, we compared the number of bases in the hit region of unigenes (contigs and singletons) to the length of the best match silkworm (Bombyx mori) protein--this "ortholog hit ratio" gives a close estimate on the amount of the transcript discovered relative to a model lepidopteran genome. For each species, we tested two assembly programs and two parameter sets; although CAP3 is commonly used for such data, the assemblies produced by Celera Assembler with modified parameters were chosen over those produced by CAP3 based on contig and singleton counts as well as ortholog hit ratio analysis. In the final assemblies, 1,413 E. propertius and 1,940 P. zelicaon unigenes had a ratio $>0.8 ; 2,866$ E. propertius and 4,015 P. zelicaon unigenes had a ratio $>05$.

Conclusions: Ultimately, these assemblies and SNP data will be used to generate microarrays for ecoinformatics examining climate change tolerance of different natural populations. These studies will benefit from high quality assemblies with few singletons (less than $26 \%$ of bases for each assembled transcriptome are present in unassembled singleton ESTs) and effective transcript discovery (over 6,500 of our putative orthologs cover at least $50 \%$ of the corresponding model silkworm gene).
\end{abstract}

\section{Background}

Although the costs of genome sequencing have declined dramatically, full genome sequencing efforts are still impractical for many nonmodel species. In such cases, transcriptome sequencing provides a greatly informative and cost effective alternative [1,2]. Expressed Sequence Tag (EST) sequencing has been used in a variety of spe-

* Correspondence: semrich@nd.edu

${ }^{1}$ Department of Computer Science and Engineering, University of Notre Dame, Notre Dame, IN, USA

Full list of author information is available at the end of the article cies for Single Nucleotide Polymorphism (SNP) discovery [3], gene discovery and annotation [4-7], and expression analysis [8-10].

While previous studies relied extensively on available genome or transcript data generated by Sanger EST sequencing, more recent results have used 454 technology to perform de novo assembly of transcriptomes. In 2008, Vera et al. sequenced ESTs of Melitaea cinxia using 454 GS20 technology, producing 108,297 contigs and singletons (ESTs which would not assemble with others), or 
"unigenes," representing an estimated $50 \%$ of the transcriptome [11]. Novaes et al. and Cheung et al. in the same year reported 454 EST assemblies for Eucalyptus grandis [12] and the plant pathogen, Pythium ultimum [13]. In 2009, Meyer et al. assembled the transcriptome of larval coral, Acropora millepora, to an average contig coverage of $5 \times$ [14], and Roeding et al. assembled the the transcriptome for the Emperor Scorpion, Pandinus imperator, to an average contig coverage of $9 \times$ [15], the highest of 454 transcriptome studies to date. These assemblies reinforce previous results that suggest 454 EST sequencing produces evenly covered transcripts with error rates mitigated by deep coverage [16].

Other published Lepidopteran EST projects include those for wing discs of adult Heliconius erato [17] and foreleg tarsi of Papilio xuthus [18]; both used Sanger based sequencing. In this paper, we present de novo larval full-body transcriptome assemblies for two butterflies: the Propertius Duskywing, Erynnis propertius (Lepidoptera: Hesperiidae), and the Anise Swallowtail, Papilio zelicaon (Lepidoptera: Papilionidae).

\section{Study Species}

E. propertius is in the family Hesperiidae (Lepidoptera), a distinct branch of the butterflies called "skippers." P. zelicaon is in the family Papilionidae (Lepidoptera) and is more closely related to all other butterflies than to any skipper. Erynnis propertius and P. zelicaon co-occur in coastal, oak (Quercus) habitats containing native wildflowers that range from Baja California, Mexico northward into southwestern British Columbia [19,20]. Erynnis propertius, an oak specialist, is restricted to this range, whereas P. zelicaon also occurs further eastward and northward in the Rocky Mountains feeding on plants in the family Apiaceae [21].

Previous studies suggested that these species are differentiated across their range with populations at the northern range boundary being diverged from more central populations [22,23]. Zakharov and Hellmann [22] suggested that these differences could allow local adaptation of northern populations, possibly to local climatic conditions, and that these local adaptations could undermine the assumption that northern populations will increase under climate warming. Hellmann et al. [24] and Pelini et al. [25] investigated this possibility with a series of translocation experiments and found greater evidence for local adaptation of northern populations in E. propertius than P. zelicaon. Pelini et al. [25] also found that climate affects fitness on alternate host plants, switching the relative value of host species under different climate treatments in P. zelicaon.

Before we performed 454 sequencing, there were no genetic data for these species with the exception of microsatellites [26,27], mitochondrial genes, and select genes identified in other Papilio (e.g. [28,29]). When compared to other transcriptomes or genomes of Lepidoptera that have been entirely sequenced, E. propertius and P. zelicaon can offer new insights into the systematics and genomics of a group that has been widely recognized for its utility in ecology and evolutionary biology [30]. In addition to advancing comparative genomics in Lepidoptera, sequencing the transcriptomes of two co-occurring species with known ecology offers many future research opportunities in ecology and ecoinformatics.

The mRNA of whole larvae were extracted for sequencing for two reasons: 1) because larvae are a key bottleneck in the population dynamics and fitness of individual butterflies [31-35] and 2) to complement previous studies by our group that measure larval fitness under differing climatic conditions $[24,25]$. The immediate aim of transcriptome sequencing was construction of a microarray enabling comparison of transcribed genes under alternate climate treatments and of populations from differing geographic locations.

Adult females were collected from 4 and 2 populations of E. propertius and $P$. zelicaon, respectively, near the latitudinal center of the species' distributions (within $50 \mathrm{~km}$ of Medford, Oregon). In total, 53 larvae of E. propertius and 61 larvae of $P$. zelicaon were pooled prior to sequencing, representing a minimum of 11 and a maximum of 48 wild mothers in E. propertius and exactly 5 wild mothers for $P$. zelicaon. To build a robust microarray for the study of larval biology, steps were taken to maximize gene discovery within the larval stage. Multiple larval instars were sampled, and individuals were exposed to a variety of stress and host plant treatments to elicit genes important in the life history of larvae (see Methods).

\section{Results}

\section{Sequencing and Assembly}

Half of one picotiter plate of a 454 FLX sequencing run generated 416,689 ESTs of E. propertius. Reads were cleaned and vector trimmed with standard SeqClean [36] protocol (see Methods). In total, 397,230 (95\%) E. propertius ESTs passed the cleaning process, with an average length 431 bp and median length 458 bp. 432,343 (95\%) ESTs of $P$. zelicaon passed cleaning, having an average length of $401 \mathrm{bp}$ and median length $422 \mathrm{bp}$. These data are publicly available at the NCBI Sequence Read Archive (see Methods).

We ran the cleaned EST datasets through CAP3 as well as the Celera Assembler, experimenting with parameter settings for each (see Methods). Using default parameter settings, CAP3 [37] produced fairly large assemblies-$24.3 \mathrm{Mbp}$ for E. propertius. Although we wish to avoid collapsing paralogs, large assemblies indicate separately assembled alleles [38]. Using custom parameters for CAP3, we reduced assembly sizes somewhat, but this still 
Table 1: Statistics for alternate assemblies. The last column indicates the average Ortholog Hit Ratio (a measure of assembly quality, see Annotation) of unigenes with hits to Bombyx mori.

\begin{tabular}{|c|c|c|c|c|c|c|}
\hline & $\begin{array}{c}\text { Contigs } \\
\text { (Coverage) }\end{array}$ & Singletons & $\begin{array}{l}\text { Assembly } \\
\text { Size }\end{array}$ & $\begin{array}{c}\text { BP in } \\
\text { Singletons }\end{array}$ & B. m. Hits & $\overline{\mathrm{OHR}}$ \\
\hline \multicolumn{7}{|c|}{ CAP3 Defaults (-p $90-h$ 20) } \\
\hline E.p. & $15,444(8.39 \times)$ & 27,516 & $24.3 \mathrm{Mbp}$ & $46.0 \%$ & 12,763 & 0.352 \\
\hline P.z. & $17,983(8.12 \times)$ & 28,884 & $25.1 \mathrm{Mbp}$ & $43.0 \%$ & 16,113 & 0.356 \\
\hline \multicolumn{7}{|c|}{ CAP3 -p 85 -h 90} \\
\hline E.p. & $\begin{array}{c}11,687 \\
(10.07 x)\end{array}$ & 20,053 & 19.1 Mbp & $42.4 \%$ & 10,183 & 0.372 \\
\hline P.z. & $13,332(9.78 \times)$ & 20,563 & $19.5 \mathrm{Mbp}$ & $38.9 \%$ & 12,740 & 0.375 \\
\hline \multicolumn{7}{|c|}{ Celera Standard } \\
\hline E.p. & $23,263(8.35 \times)$ & 13,444 & $20.6 \mathrm{Mbp}$ & $21.3 \%$ & 11,625 & 0.384 \\
\hline P.z. & $25,822(8.05 \times)$ & 21,397 & $22.9 \mathrm{Mbp}$ & $24.4 \%$ & 16,755 & 0.398 \\
\hline \multicolumn{7}{|c|}{ Celera Final } \\
\hline E.p. & $\begin{array}{c}17,110 \\
(10.05 \times)\end{array}$ & 10,934 & 16.4 Mbp & $21.3 \%$ & 9,393 & 0.402 \\
\hline P.z. & $19,110(9.63 \times)$ & 18,847 & $18.5 \mathrm{Mbp}$ & $25.9 \%$ & 12,485 & 0.412 \\
\hline
\end{tabular}

produced a large percentage of singletons (Table 1). Celera Assembler [39] produced a 20.6 Mbp assembly for $E$. propertius using the recommended settings for 454 FLX Titanium reads [40]. Using custom settings with the Celera Assembler produced assemblies with the smallest overall assembly size and highest average ortholog hit ratio, a measure of assembly quality (Table 1, see Annotation). The size of this final assembled E. propertius transcriptome $(16.4 \mathrm{Mbp})$ is similar to that previously produced for the related butterfly species $M$. cinxia (approximately 16.1 Mbp [11]). While the final P. zelicaon assembly is somewhat larger $(18.5 \mathrm{Mbp})$, differences in assembly size between assemblers and parameter sets were similar to those seen for E. propertius.

The custom Celera assembly for $E$. propertius resulted in 17,110 contigs and 10,934 singletons, for a total of 28,044 unigenes. Both the average contig length and average singleton length are noticeably larger than previous studies [11-14] at 753 bp and 324 bp, respectively. Cleaned P. zelicaon ESTs assembled into 19,110 contigs (average length $714 \mathrm{bp}$ ) and 18,847 singletons (average length $258 \mathrm{bp}$ ). The larger number of unassembled singletons for $P$. zelicaon may be due to mitochondrial rRNA sequences (see Clustering). Figure 1 shows the distribu- tions of contig and singleton lengths for both species; other detailed assembly statistics also are found in Table 2 .

Average (median) contig coverage was $10 \times(3.3 \times)$ for $E$. propertius and $9.6 \times(3.5 \times)$ for $P$. zelicaon. Figure 2 shows the contig coverage distributions for the two transcriptomes and the average sequence length for contigs within each coverage bin on a log scale. As expected and as found in previous studies $[14,41]$, there was a positive correlation between contig length and the number of reads incorporated (data not shown). Figure 2 also shows that contigs with very high coverage (greater than $100 \times$ ) tend to be shorter in length.

\section{Annotation}

\section{Bombyx mori, Gene Assembly Completeness}

We compared the unigene sets to the predicted protein database for Bombyx mori, the silkworm, for which full genome data are available (GLEAN produced consensus gene set, SilkDB v2.0 [42]). This reference dataset contains 14,623 predicted $B$. mori proteins. Of the $28,044 E$. propertius unigenes, 9,393 had BLASTX [43] (using a 1e5 cutoff) hits to 7,866 unique $B$. mori predicted proteins. 5,289 unigenes hit more than one $B$. mori protein (aver- 


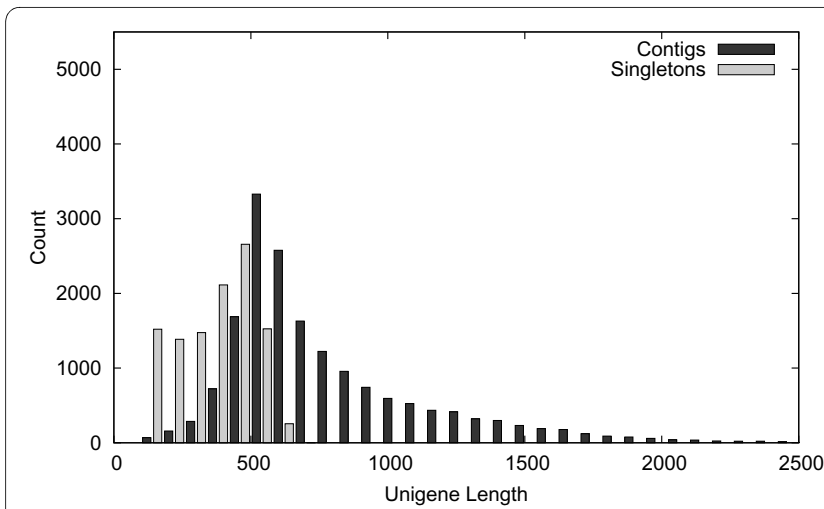

(a)

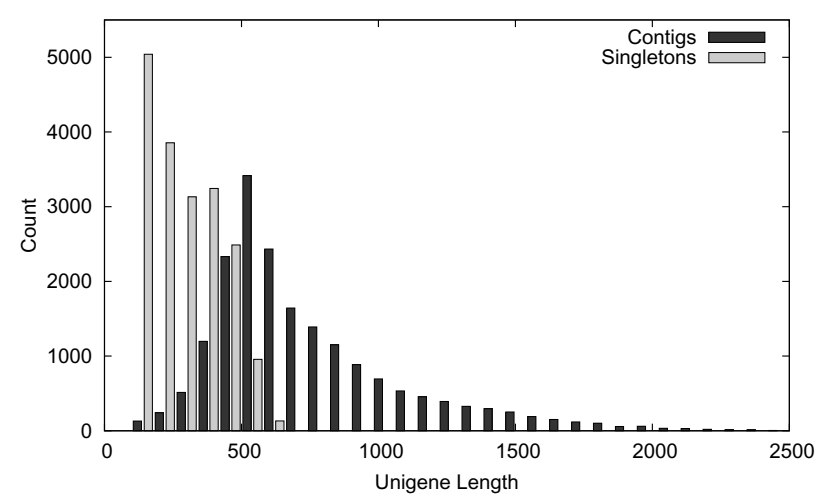

(b)

Figure 1 Distributions of contig and singleton lengths for E. propertius (a) and P. zelicaon (b). Contigs longer than 2,500 bp ( $n=34$ for E. propertius, max length $3,737 \mathrm{bp}, n=26$ for $P$. zelicaon, max length 3,621 bp) are not shown.

age, 8.9, median, 2.0, amongst unigenes hitting at least one protein). $5,449 \mathrm{~B}$. mori proteins were hit by more than one unigene (average, 10.6, median, 3.0, amongst proteins having at least one hit). Of the 37,957 P. zelicaon unigenes, 12,485 hit 8,359 unique $B$. mori predicted proteins; 6,518 hit more than one protein (average, 8.8, median, 2.0 ), and 5,883 proteins were hit by more than one unigene (average, 13.1, median, 3.0). Figure 3 shows the distribution of 24 categories for gene ontology terms, each categorized into three higher level categories, associated with the unigenes and the B. mori dataset (see Methods).

For the purposes of this study, we consider each unigene and its best B. mori BLASTX hit to be orthologs, and we consider the hit region in the unigene to be a conservative estimator of the "putative coding region." Thus, we can compute the percentage of a unigene found by dividing the length of the putative coding region by the total length of the ortholog. This ratio, which we call the "ortholog hit ratio," is described in Figure 4. The assumption is that the unigene and its best hit are orthologs and not paralogs or some other mis-association. Using the conservative, BLAST based annotation to find putative coding regions, as opposed to non-comparative methods such as ESTScan [44], ensures that hit ratios are not overestimated.
The ortholog hit ratio gives an estimate on the amount of a transcript contained in each unigene. If there are relative insertions in best hit $B$. mori proteins, this will tend to lower ortholog hit ratios, whereas relative insertions in unigenes will artificially inflate ortholog hit ratios. Ortholog hit ratios greater than 1.0 likely indicate large insertions in unigenes.

Figures 5(a) and 5(b) show ortholog hit ratio in terms of assembly coverage of unigenes (which is 1.0 for singletons). For E. propertius contigs with less than the median assembly coverage of $3.3 \times$, the average ortholog hit ratio was 0.35 . For those with greater than median coverage, the average ratio was 0.56 . The corresponding averages for $P$. zelicaon were 0.34 and 0.55 , respectively. Thus, completeness of unigene assembly is partially governed by assembly coverage as expected.

Figures 5(c) and 5(d) relate ortholog hit ratio to the length of the B. mori ortholog. As found in other studies [11], completeness of gene discovery decreases as length of the gene increases. Vertical tracks in figures 5(c) and 5(d), comprised mostly of singletons, likely indicate regions of the genome that failed to assemble (see Clustering). Finally, figures 5(e) and 5(f) show the overall distributions of ortholog hit ratios for contigs and singletons. Overall, 1,413 of the 9,393 E. propertius unigenes having a hit to $B$. mori had ratio $>0.8$, and 2,866 had

Table 2: EST and final assembly statistics.

\begin{tabular}{|c|c|c|c|c|c|c|c|c|c|c|c|}
\hline & \multicolumn{2}{|c|}{ Uncleaned Reads } & \multicolumn{2}{|c|}{ Cleaned Reads } & \multicolumn{2}{|c|}{ Contigs } & \multicolumn{2}{|c|}{ Singletons } & \multicolumn{3}{|c|}{ Unigenes } \\
\hline & $n$ & $\bar{x} \mathbf{b p}$ & $n$ & $\bar{x} \mathbf{b p}$ & $n$ & $\bar{x} \mathbf{b p}$ & $n$ & $\bar{x} \mathbf{b p}$ & $n$ & $\bar{x} \mathbf{b p}$ & $\begin{array}{c}\text { Median } \\
\text { bp }\end{array}$ \\
\hline E.p. & 416,689 & 424 & 397,230 & 431 & 17,110 & 753 & 10,934 & 324 & 28,044 & 586 & 502 \\
\hline P.z. & 455,040 & 398 & 432,343 & 401 & 19,110 & 714 & 18,847 & 258 & 37,957 & 488 & 414 \\
\hline
\end{tabular}




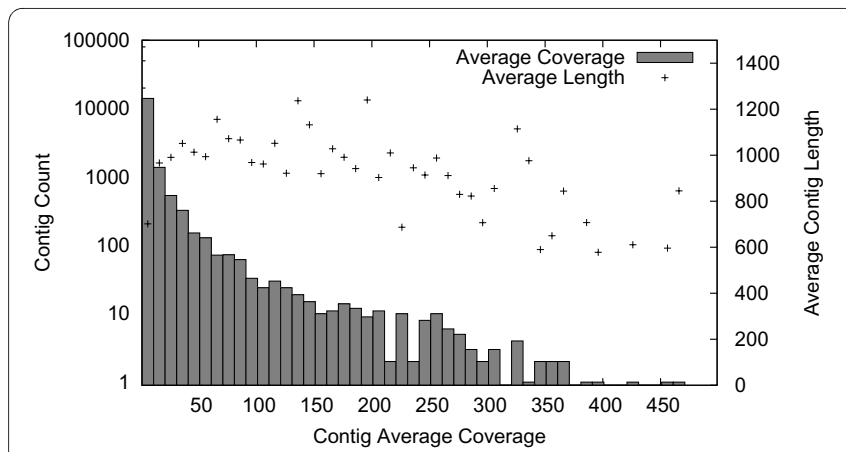

(a)

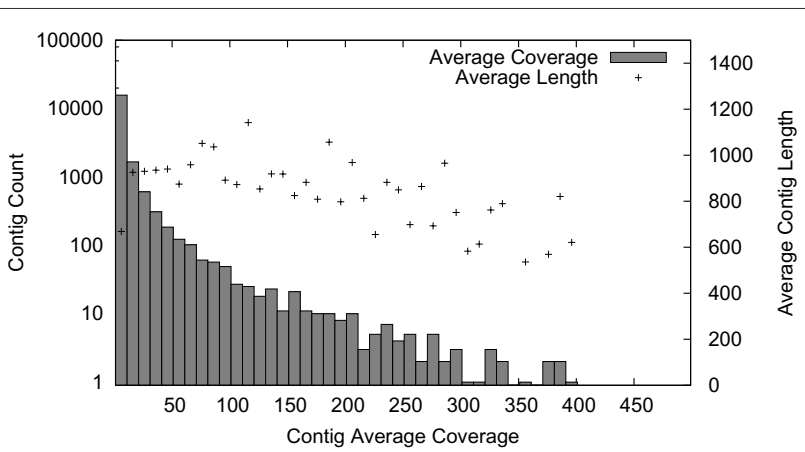

(b)

Figure 2 Distribution of average contig read coverage for E. propertius (a) and $P$. zelicaon (b). On the $x$ axis, contigs are grouped by average read coverage. On the $y$ axis, the bars show the number of contigs in each coverage bin, and the points show the average length of contigs in each coverage bin.

ratio $>0.5$. Of the $12,485 P$. zelicaon unigenes with $B$. mori hits, 1,940 had ratio $>0.8$ and 4,015 had ratio $>0.5$.

\section{Other Lepidoptera and Insecta}

We also compared unigene sets to protein databases for Drosophila melanogaster (FlyBase, r5.22 [45]), containing 21,783 sequences and Heliconius erato (ButterflyBase, retrieved April, 2009 [46]), containing 8,790 sequences. Drosophila melanogaster proteins represent a well annotated insect transcriptome, and the $H$. erato database represents protein predictions based on tissue-specific Sanger EST data obtained from the wing discs of adults $[46,47]$. While this tissue-specific dataset is not as complete as the $D$. melanogaster protein dataset, comparison to the more related $P$. zelicaon and less closely related $E$. propertius reveals interesting differences.

5,688 E. propertius unigenes had BLASTX hits (1e-5 cutoff) to $H$. erato proteins. 7,497 had hits to D. melano- gaster proteins. 11,082 P. zelicaon unigenes hit $H$. erato proteins, a much larger percentage (29.1\% versus $20.2 \%)$, and 9,689 hit D. melanogaster.

Figure 6 shows the number of unigenes with hits to one or more of the three protein databases. Venn diagram areas are scaled to represent percentages of the unigene sets. Although both species had a large number of hits to $H$. erato, this database is comparatively small as indicated by Figure 6.

The bars in Figure 6 show the relative proportion of high coverage contigs (greater than median coverage), low coverage contigs (less than median coverage), and singletons for each area in the Venn diagram.

Unigenes that hit to all three databases tend to have high coverage, while those that only hit the most related species (H. erato) tend to have low coverage or are singletons. 7,266 E. propertius singletons and 10,462 contigs

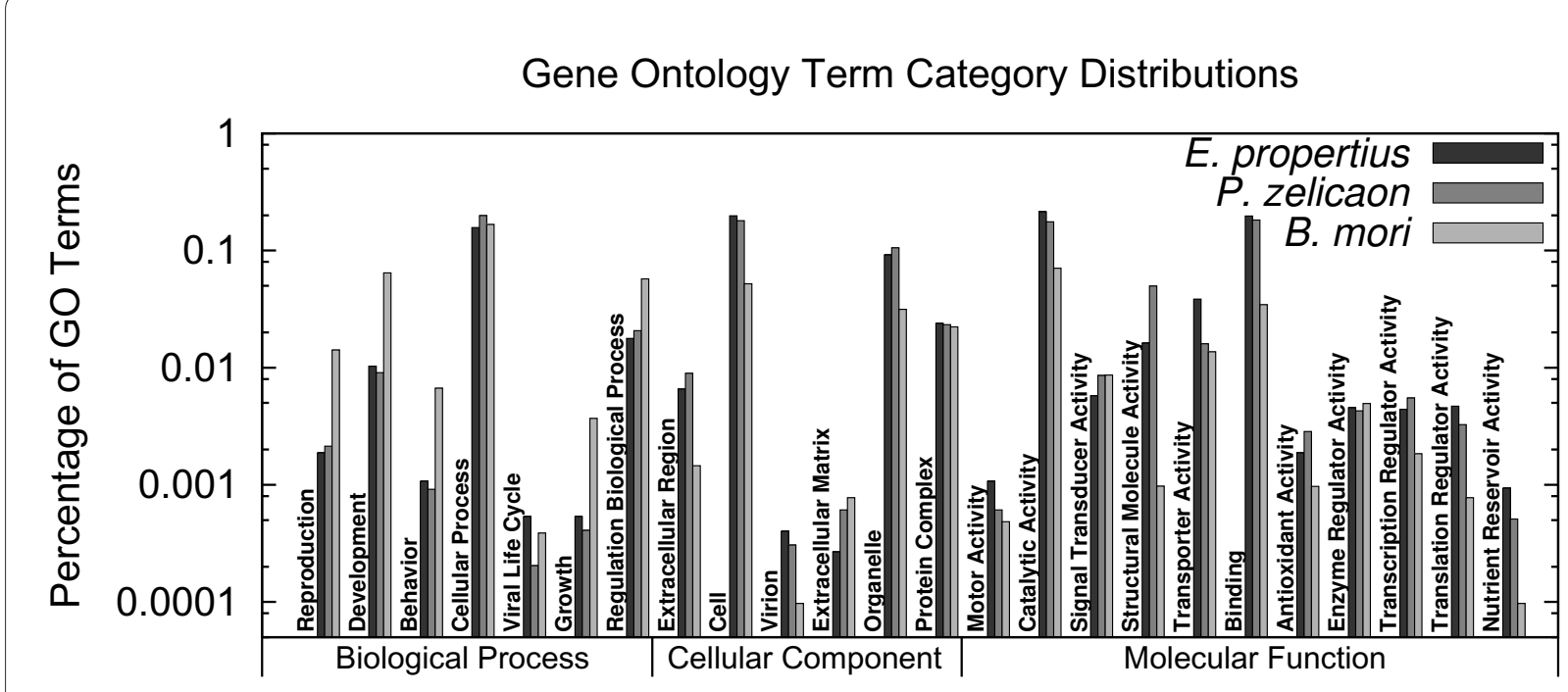

Figure 3 Distribution of Gene Ontology terms for E. propertius, P. zelicaon, and B. mori (see Methods). 


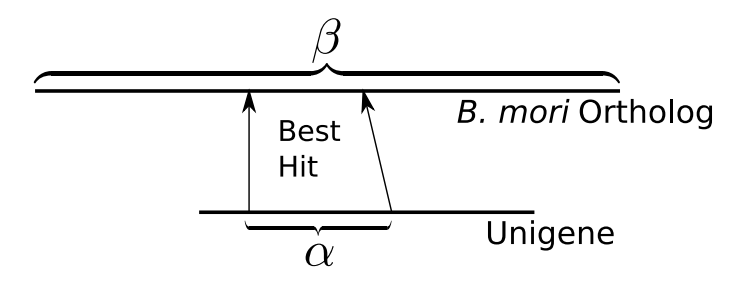

"Ortholog Hit Ratio" = (\#Bases in $\alpha) /(\#$ Bases in $\beta)$

Figure 4 The ortholog hit ratio describes the percentage of an ortholog "found" in a unigene by dividing the number of non-gap characters in the query hit by the length of the subject

with average coverage of $8 \times$ had no hits to these protein databases. For P. zelicaon, 9,871 singletons and 10,083 contigs with average coverage of $6.6 \times$ had no hits.

We also compared the unigene sets to recently sequenced whole larval Melitaea cinxia ESTs [11] and foreleg tarsi Papilio xuthus ESTs [18]. Although there is no protein, assembly, or annotation information publicly available for these datasets, we expect some similarity given phylogenetic distance. 9,979 E. propertius unigenes had TBLASTX hits (1e-5 cutoff) to 76,809 unique $M$. cinxia ESTs. 16,780 P. zelicaon unigenes had hits to 82,906 unique $M$. cinxia ESTs (out of 595,541). As expected, many more $P$. zelicaon unigenes had hits to $P$. xuthus--4,511 E. propertius unigenes hit 8,329 unique $P$. xuthus ESTs, while 16,492 P. zelicaon unigenes hit 13,043 unique $P$. xuthus ESTs (out of 16,802).

\section{Clustering}

To test whether incomplete assembly could account for the large proportion of singletons that hit only $H$. erato (Figure 6), we aggressively clustered unigenes by creating "association graphs" of best hits of unigenes to unigenes, unigenes to $H$. erato proteins, and unigenes to B. mori proteins. Unigenes in the same connected component were considered a cluster (see Methods). E. propertius unigenes produced 20,667 clusters, 19,037 of which contained only a single unigene. The largest E. propertius cluster contained 485 singletons (434 having hits only to H. erato) and 65 contigs (all of which hit only H. erato). $P$. zelicaon unigenes produced 21,530 clusters, 19,395 containing only a single unigene. For this species, a single very large cluster of 6,124 singletons and 209 contigs was produced. As with E. propertius, most of these singletons $(4,832)$ and contigs $(180)$ had hits only to H. erato proteins.

Most of the unigenes in the very large cluster for $P$. zelicaon were similar, though it appeared that a large amount of sequence diversity prevented their assembly into contigs. As mitochondrial genomes are frequently diverse in populations [22], we compared unigenes to Papilio xuthus mitochondrial genes and ribosomal RNAs (GenBank: EF621724). Of the 6,333 unigenes in the largest $P$. zelicaon cluster, 3 had a BLASTN hit to P. xuthus mitochondrial genes ( $e<1 \mathrm{e}-5$ cutoff) and 5,995 hit ribosomal RNA. We also identified 59 unigenes not present in the largest cluster that hit mitochondrial genes, and 1,275 that hit ribosomal RNA. All but a few ribosomal hits were to the $16 \mathrm{~S}$ ribosomal RNA. Similar analysis of E. propertius unigenes revealed 43 hits to $P$. xuthus mitochondrial genes and 50 hits to ribosomal RNA, none of which occurred in the largest cluster.

To validate clustering results, we used TBLASTX $(e<$ 1e-5 cutoff) to search for five single-copy genes from $B$. mori: CAD carbamoylphosphate synthase domain (GenBank:EU032656), PGD 6-phosphogluconate dehydrogenase (GenBank:NM 001047060), AATS alanyl-tRNA synthetase (GenBank:M55993), SNF sans fille (GenBank:DQ202313), and TPI triosephosphate isomerase (GenBank:NM 001126258) [48]. Because these genes are single copy, a correct clustering should identify unigenes orthologous to them as being related. PGD and SNF each had hits to a single contig in the E. propertius unigene set (covering $30 \%$ and $52 \%$ of PGD and SNF, respectively); neither of these contigs were clustered with any other unigenes. The TPI gene had a hit to a contig (covering $56 \%$ of TPI) that also was clustered with one other singleton. The other genes, CAD and AATS, had no hits in the E. propertius unigene set.

For P. zelicaon, the PGD gene had hits to three contigs (together covering 9\% of PGD); these were clustered together along with one other singleton. The TPI gene hit a single contig (covering $65 \%$ of TPI) that also was clustered with one other contig. The AATS gene hit three contigs representing two full clusters (covering $45 \%$ of AATS). The SNF and CAD genes had no hits to the $P$. zelicaon unigene set.

To investigate the absence of CAD and AATS for $E$. propertius and CAD and SNF for P. zelicaon, we searched for evidence of these genes in the $M$. cinxia EST dataset [11]. Of the 595,541 uncleaned $M$. cinxia ESTs, 35 hit SNF, 75 hit AATS, and 1 hit CAD. Thus, although these genes appear to be expressed in a lepidopteran larval transcriptome, they appear to be present at low levels in EST collections, particularly for CAD.

\section{Metatranscriptomic Contamination}

Because material was sampled from whole larvae, we expect some unigenes to represent species other than $E$. propertius and P. zelicaon. Of the 15,555 E. propertius unigenes with no hits to D. melanogaster, B. mori, $H$. erato, $M$. cinxia, or P. xuthus, 90 had hits to other Metazoa (63 Insecta; see Methods). 69 E. propertius unigenes hit Viridiplantae, 16 hit Bacteria, 5 hit Fungi, and 12 unigenes hit species in various other kingdoms.

Of the 12,941 P. zelicaon unigenes with no hits to the five species mentioned above, 165 hit Metazoa (132 Insecta). 56 hit Viridiplantae, 22 hit Bacteria, 41 hit Fungi, 


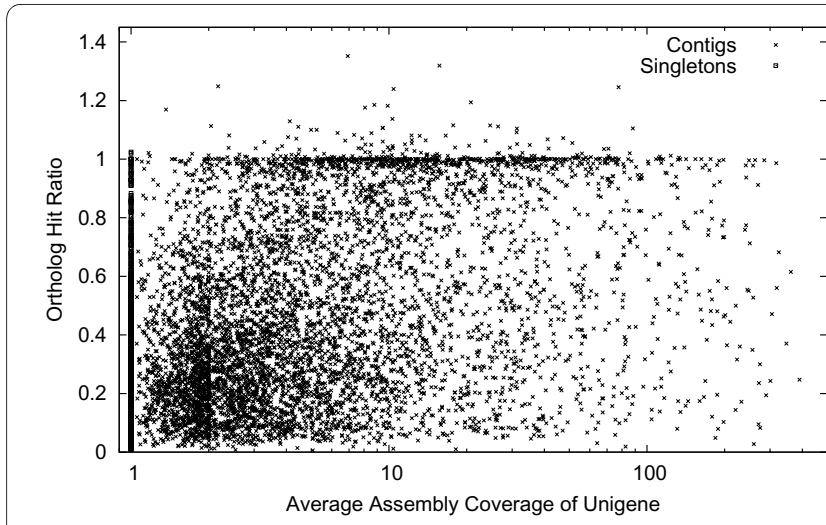

(a)

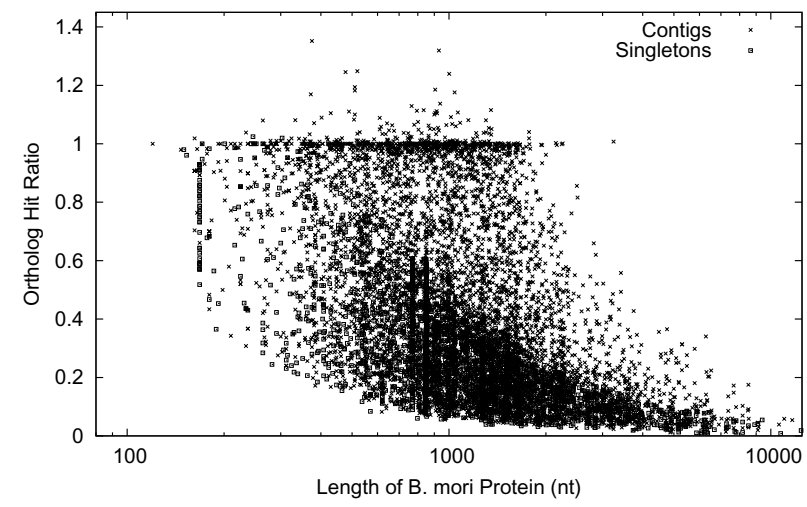

(c)

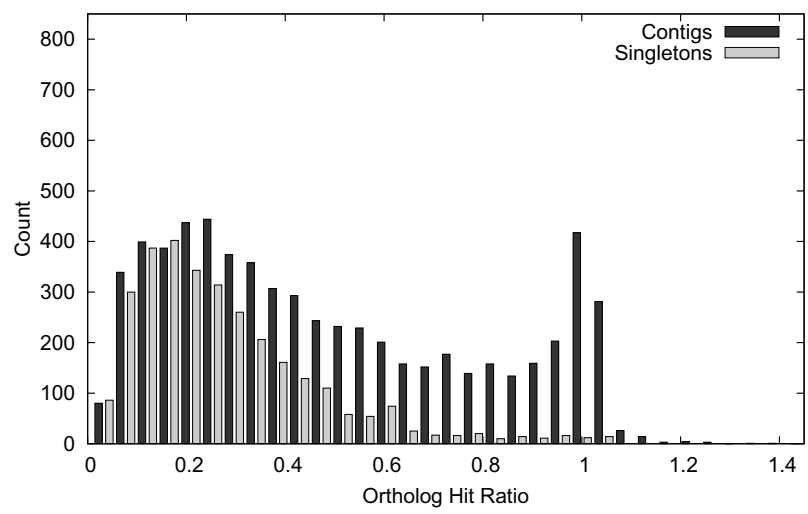

(e)

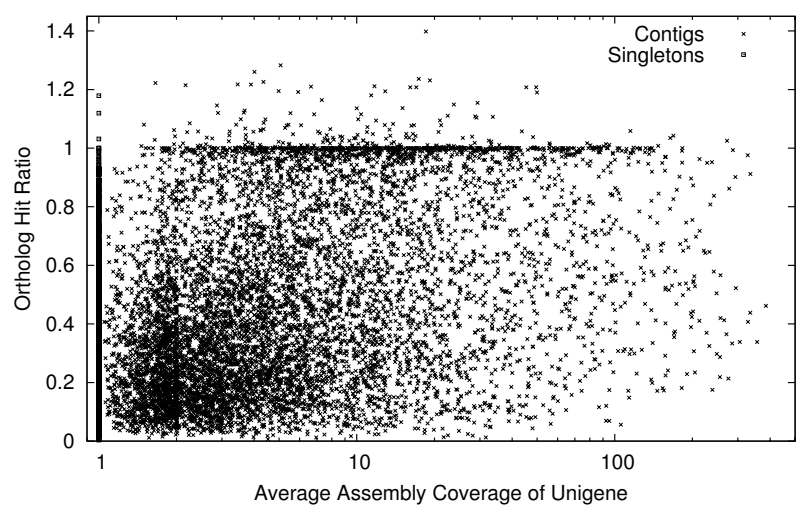

(b)

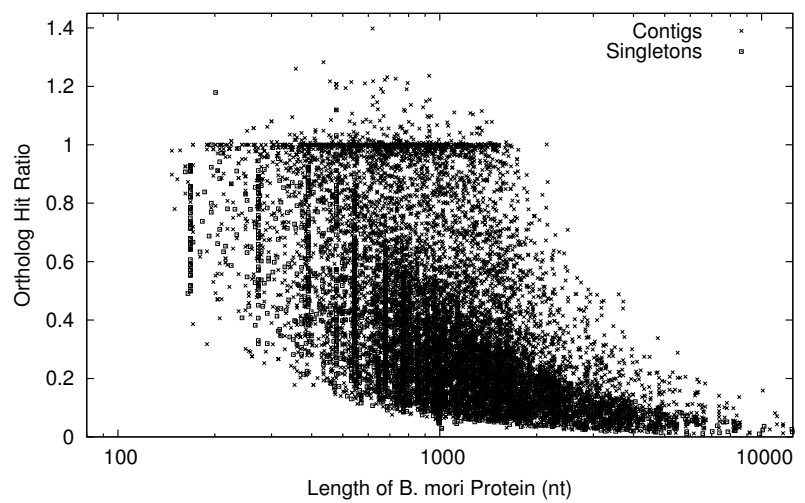

(d)

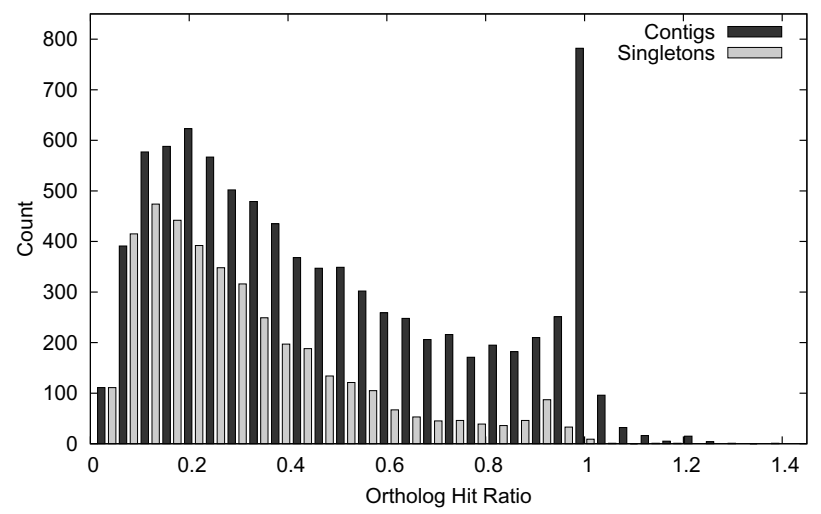

(f)

Figure 5 Relationship between "ortholog hit ratio" (see Figure 4) and assembly coverage (a,b) as well as B. mori ortholog length (c, d). Figures on the left refer to E. propertius, figures on the right refer to $P$. zelicaon. Where this ratio is 1.0 , the gene is likely fully assembled. Ratios greater than 1.0 can indicate insertions in unigenes. Overall distributions of ortholog hit ratios for contigs and singletons also shown (e,f).

and 12 hit other kingdoms. For both species, the bacterial hits included one singleton which hit to Wolbachia (of $D$. melanogaster, e-value 2e-6 for E. propertius and 2e-15 for P. zelicaon).

\section{Genetic Diversity}

\section{SNP Detection}

Single Nucleotide Polymorphisms (SNPs) were identified by analyzing the multiple alignments produced during the assembly process using both a "loose" criterion to maximize the discovery of rare alleles, and a "strict" criterion to minimize the possibility of false positives due to sequencing error (see Methods).

Table 3 shows SNP counts and other statistics using these two criteria. For both criteria, E. propertius had a slightly higher percentage of transversions than $P$. zelicaon ( $45 \%$ and $44 \%$ vs. $43 \%$ and $42 \%$, respectively). These 


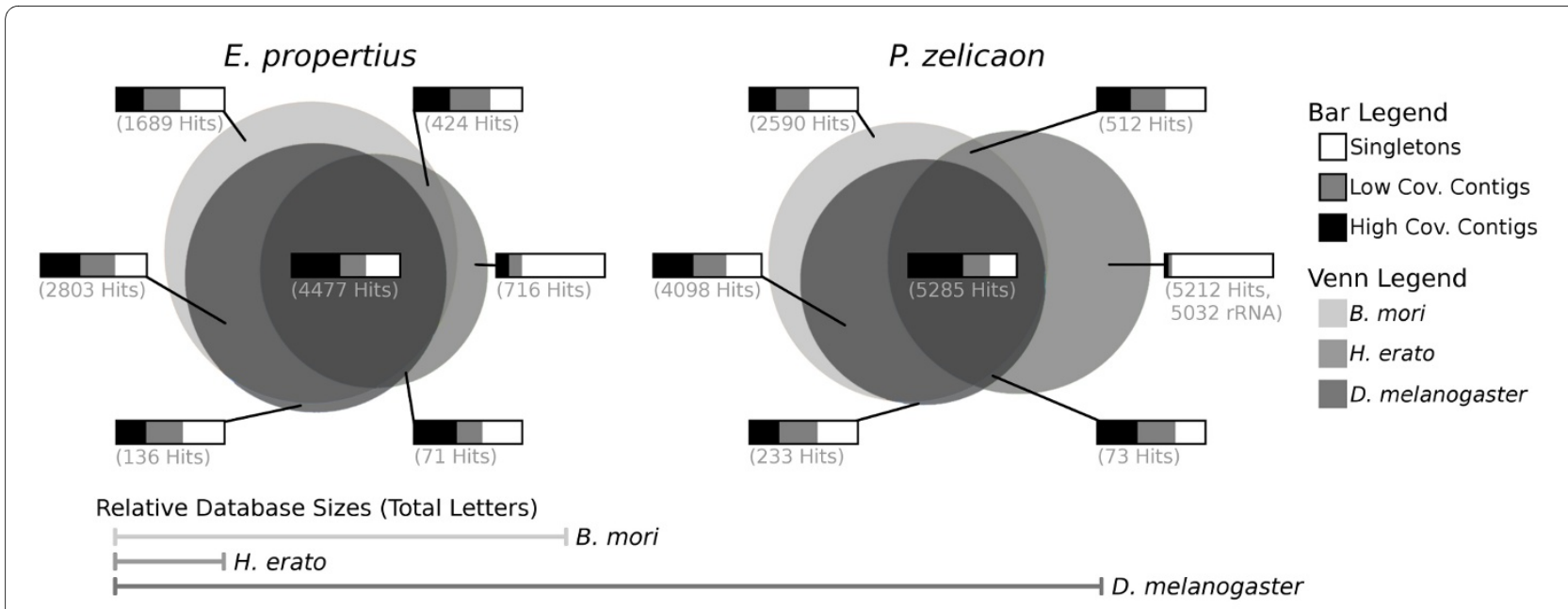

Figure 6 BLAST unigene results relative to protein databases for B. mori, H. erato, and D. melanogaster. Note that these databases are not of equal size, and that the protein database for $\mathrm{H}$. erato represents tissue-specific samples of adult wing discs $[46,47]$. Bars show the relative proportion of high coverage contigs (greater than median coverage), low coverage contigs (less than median coverage), and singletons for each Venn diagram area. Many of the hits for P. zelicaon unigenes which hit only to H. erato also hit to the mitochondrion 165 ribosomal RNA.

transversion percentages are between those found for $B$. mori, 37.5\% [49], and D. melanogaster, 51.9\% [50].

For E. propertius, strict criterion SNPs were found in 6,298 contigs, comprising $6.11 \mathrm{Mbp}$ of sequence. Thus, we estimate at least $36,014 \mathrm{SNPs}$ in $6.11 \mathrm{Mbp}$ or 5.89 SNPs per 1,000 bases for E. propertius. Similar calculations for P. zelicaon discover at least 9.28 SNPs per 1,000 bases. In comparison, Vera et al. estimated 12.6 SNPs per 1,000 bases in probable coding regions of the $M$. cinxia transcriptome using similar source data and similar conservative criteria for identifying SNPs [11].

We also can label SNPs that appear in putative coding regions (as found by BLAST against B. mori, see Annotation) as non-synonymous or synonymous (Table 3 ). Strict criterion SNPs occurred in 2,067 putative E. propertius coding regions, representing $1.52 \mathrm{Mbp}$ of sequence. Thus, we estimate at least $1,648 \times 10^{3} / 1.52 \times 10^{6} \approx 1.08$ non-synonymous SNPs and $8,273 \times 10^{3} / 1.52 \times 10^{6} \approx 5.44$ synonymous SNPs per 1,000 base pairs in coding regions of the E. propertius transcriptome. For P. zelicaon, strict SNPs occurred in 3,384 putative coding regions representing 2.19 Mbp of sequence, for an estimate of 2.09 non-synonymous and 8.56 synonymous SNPs per 1,000 base pairs in coding regions of the $P$. zelicaon transcriptome.

\section{Celera Variant Detection}

Recent versions of the Celera assembler cluster co-occurring SNPs and indels together into "variants"--polymorphisms that may include more than a single nucleotide but yet are not large enough to be considered haplotypes.

Table 3: SNP discovery statistics. Loose criterion: non-gap consensus in the multiple alignment, minority nucleic allele found in at least two ESTs. Strict criterion: non-gap consensus in the multiple alignment, minority nucleic allele found in at least $\mathbf{2 5 \%}$ of ESTs covering the position, at least $\mathbf{6 x}$ coverage at the position. Non-synonymous and synonymous SNPs were counted via best BLAST hits to $B$. mori.

\begin{tabular}{|c|c|c|c|c|c|c|}
\hline & SNPs & Transversions & Transitions & Non-Syn & Syn & Contigs With SNPs \\
\hline \multicolumn{7}{|c|}{ Loose SNP Criterion } \\
\hline E.p. & 94,783 & 42,719 & 52,064 & 5,341 & 20,520 & 8,042 (7.52 Mbp) \\
\hline P.z. & 127,004 & 54,934 & 72,070 & 10,193 & 36,170 & 8,888 (7.97 Mbp) \\
\hline \multicolumn{7}{|c|}{ Strict SNP Criterion } \\
\hline E.p. & 36,014 & 15,895 & 20,119 & 1,648 & 8,273 & 6,298 (6.11 Mbp) \\
\hline P.z. & 62,655 & 26,545 & 36,110 & 4,527 & 18,509 & 7,223 (6.75 Mbp) \\
\hline
\end{tabular}


These variants also inform the Celera assembly process, so that chimeric contigs containing nearby allele combinations not found in nature are avoided [51]. By default, variants are identified by grouping polymorphisms together so long as a stretch of at most 11 non-polymorphic sites occur between them, and each allele is supported by at least two reads. Quality values are also used.

Not counting single nucleotide length variants (SNPs surrounded by at least 11 non-polymorphic sites), the assembly produced 6,775 variant regions in 3,697 E. propertius contigs, with an average length of 3.43 bp (maximum $41 \mathrm{bp}$ ). The average number of variants per region was 2.93 , with a maximum of 17 , consistent with the maximum number of genotypes sequenced. In this case, the largest variant region of $41 \mathrm{bp}$ was also the region with the most number of variants, 17 . The vast majority of ESTs (428/475) in this region supported a single variant, with the second most frequent variant occurring in only 9 ESTs.

For P. zelicaon, there were 5,636 variant regions in 3,494 contigs, with average length $2.90 \mathrm{bp}$ (maximum $27 \mathrm{bp}--\mathrm{a}$ large indel with only two variants). The average number of variants per region was 2.42 , with a maximum of 12 (a region of length $6 \mathrm{bp}$ ). This large number of variants is inconsistent with the fact that only 10 genotypes were sequenced, and may indicate paralog collapse during assembly or sequencing error. Alternatively, it is possible that one or more of the P. zelicaon mothers was fertilized by more than one male, resulting in more genotypes present in the data than female lines [52,53]. This is the only variant region for $P$. zelicaon with more than 10 variants.

\section{$\beta$ Parameter}

Because ESTs were sequenced from a number of genotypes and because assembly coverage varies among contigs, standard measures of nucleotide diversity such as $\theta$ [54] can not be calculated. Instead, we consider a relative measure of nucleotide diversity $\beta_{t}$ developed by Novaes et al. [12], defined for contigs with average coverage at least $2 \times$. (Note that even contigs with $2 \times$ average coverage can have regions of locally high coverage where SNPs can be found.) However, for all that follows, we compute $\beta$ statistics only for those contigs with at least $6 \times$ average cover- age to avoid biases caused by contigs that represent diverse sequences but are expressed at low levels. For contigs that also have a B. mori best hit (see Annotation), we can compute $\beta_{n}$, a diversity estimate for non-synonymous sites, and $\beta_{s}$, a diversity estimate for synonymous sites [12]. $\beta_{t}, \beta_{n}$, and $\beta_{s}$ are formally defined as follows:

$$
\begin{aligned}
& \beta_{t}=\frac{S_{t}+1}{L_{t} H\lfloor D\rfloor-1}, \\
& \beta_{n}=\frac{S_{n}+1}{L_{c} H\lfloor D\rfloor-1}, \\
& \beta_{s}=\frac{S_{s}+1}{L_{c} H\lfloor D\rfloor-1} .
\end{aligned}
$$

In the above, $S_{t}$ is the number of SNPs in the contig (using the strict SNP criterion; see Annotation), $S_{n}$ is the number of non-synonymous SNPs in BLAST annotated putative coding regions, $S_{s}$ is the number of synonymous SNPs in putative coding regions, $L_{t}$ is the total length of the contig, $L_{c}$ is the length of the putative coding region, $D$ is the average coverage depth, and $H_{n}$ is the $n^{\text {th }}$ harmonic number. Table 4 shows average and median values of $\beta_{t}, \beta_{n}$ and $\beta_{s}$ amongst contigs with at least $6 \times$ coverage for both species.

Novaes et al. note that because $\beta$ statistics are conditioned on coverage depth rather than the actual number of haplotypes sampled, care must be taken in comparing to more traditional diversity estimates such as $\theta$ [12]. However, these statistics do enable the study of relative genetic diversity within each transcriptome [12], and may speak to comparative diversity estimates for E. propertius and $P$. zelicaon if allele sample rates are equal (which is not the case; nevertheless, see Discussion).

The average coverage for $E$. propertius contigs in the top $1 \%$ of $\beta_{t}$ was relatively low at $8.8 \times$ (even considering the fact that this is computed only over contigs with at least $6 \times$ coverage). The average $\beta_{t}$ for $E$. propertius contigs in the top $1 \%$ of coverage also was low at $0.68 \times 10^{-3}$. For P. zelicaon, average coverage in the top $1 \%$ of $\beta_{t}$ was

Table 4: $\boldsymbol{\beta}$ parameter statistics for contigs. Statistics for $\boldsymbol{\beta}_{t}, \boldsymbol{\beta}_{s}$, and $\boldsymbol{\beta}_{n}$ are over all contigs for which those values are individually defined and contig average coverage is at least $6 \times$. SNPs used in calculation are identified using the strict SNP criterion (see Annotation).

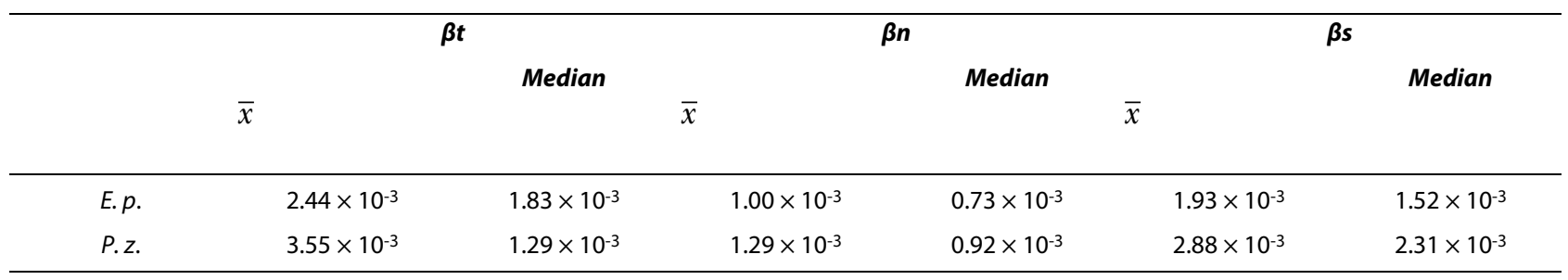


$10.64 \times$, and the average $\beta_{t}$ in the top $1 \%$ of coverage was $1.29 \times 10^{-3}$.

Thus, for both species, very diverse contigs tend to have less than or near average coverage; conversely, highly covered contigs have low diversity. In the presence of large scale paralog collapse, we would expect to see many contigs with high coverage and high $\beta$, which we have not found.

\section{Discussion}

For E. propertius, the large sequences produced by the 454 FLX Titanium allowed for the formation of a 14.6 Mbp assembly from $176 \mathrm{Mbp}$ of EST sequence, with average contig coverage of $10 \times$ and average contig length of $753 \mathrm{bp}$. Similar results were obtained for $P$. zelicaon.

Comparisons to Bombyx mori suggest that our final assemblies are of high quality. Because $\beta_{t}$ was generally low for highly covered contigs, and nearly all variant regions had fewer variants than the number of genotypes sequenced, we see little evidence for over-assembly and paralog collapse. Further, the fact that amongst the assemblies tested we have not seen a point of diminishing returns in terms of average ortholog hit ratio (Table 1) suggests that even more aggressive assemblers may produce more accurate assemblies for such diverse datasets.

Clustering results and comparison to the $P$. xuthus mitochondrial genome indicate the presence of ribosomal RNA in at least the P. zelicaon dataset. Although mitochondrial genes (e.g. ND5 and ATP6) are polyadenylated and appropriately found in our datasets, ribosomal RNAs are not, and hence should be considered contamination. While such unigenes can easily be filtered after assembly, the fact that many of these were clustered via hits to a protein predicted dataset (for $H$. erato) highlights the need for well annotated and curated reference datasets.

Clustering results also reveal that greater than $90 \%$ of unigenes had no similarity with other unigenes, indicating thorough assemblies. We searched for five single copy genes present in B. mori [48]. For those E. propertius homologues we found, assembly of unigenes was fairly complete (only one contig associated with each gene) and clustering was accurate (only one cluster contained an extra singleton). For P. zelicaon, assembly was less complete (multiple contigs per gene), although in only one case were contigs split across two clusters. Coverage of found genes was around 50\%, with the exception of the low coverage for PGD. For both organisms, no evidence was found for two of the five genes; based on similar analysis for M. cinxia ESTs, this appears to be the result of low expression in larval samples.

Determining the breadth of coverage of the transcriptomes is difficult, given how little is known about butter- fly genomes. Unigenes for both E. propertius and $P$. zelicaon hit $\sim 8 \mathrm{~K}$ (of $\sim 14.5 \mathrm{~K}$ total) unique $B$. mori predicted proteins. For both species, at least $9 \mathrm{~K}$ unigenes hit one of B. mori, H. erato, or D. melanogaster. Excluding the largest clusters, these hits were distributed roughly evenly between high and low coverage contigs and singletons, supporting previous studies suggesting that singletons and low coverage contigs are biologically valuable [14]. Gene ontology term analysis reveals that 24 high level categories are present for both species in levels similar to that for B. mori. Thus, although we cannot speculate on how many transcripts exist in these transcriptomes, they appear to be sampled broadly.

As expected in whole larval samples, we identified unigenes representative of plants, bacteria, fungi, and other non-lepidopteran sources [11]. Interestingly, a single EST from both species hit to Wolbachia, a symbiotic bacteria known to affect population dynamics and hypothesized to be present in E. propertius populations [23].

Vera et al. compared unigene length to length of the best hit protein to estimate completeness of transcript discovery [11]. Unfortunately, this also includes untranslated regions in unigenes, artificially inflating the desired measure. Our alternative, the ortholog hit ratio, provides a more conservative estimate of the effectiveness of gene discovery and speaks to assembly quality. Greater than $5 \%$ of unigenes had a ratio $>0.8$ and greater than $10 \%$ of unigenes had a ratio $>0.5$ for both species. We conclude that at least ten percent of our putative $B$. mori orthologs capture approximately $50 \%$ of their corresponding silkworm genes.

The effects of alternative splicing on the ortholog hit ratio depend on the abilities of the assembler, as well as on whether the reference $B$. mori protein set contains orthologs to alternatively spliced transcripts. Since many assemblers split contigs at ambiguous or repetitive regions [55], alternative splicing will likely result in a low ortholog hit ratio for the alternative version, even if the alternative ortholog exists in the reference dataset. If the alternative ortholog does not exist in the reference dataset, either the alternative segment will match a subregion of the original transcript form (resulting in a low ortholog hit ratio), or there may be no hit at all (resulting in an undefined ortholog hit ratio). Since these issues serve to reduce ortholog hit ratios rather than inflate them, the conservativeness of the ortholog hit ratio approach is preserved.

While estimating genetic diversity accurately (e.g. computing $\theta$ [54]) is difficult given the essentially unknown number of natural alleles contributing to the population sample for each contig, some relative comparisons between species should be possible. For example, using a SNP calling criterion similar to our strict criterion and a source dataset similar to ours, Vera et al. estimate 12.6 
SNPs per 1,000 base pairs in the $M$. cinxia transcriptome [11], whereas we estimate $5.89 \mathrm{SNPs} / 1,000$ bp for E. propertius and $9.28 \mathrm{SNPs} / 1,000 \mathrm{bp}$ for P. zelicaon.

While Novaes et al. caution against comparing $\beta$ diversity estimates across assemblies--as they depend on sequencing depth, SNP calling criteria, and other factors-the E. propertius and P. zelicaon datasets were collected and processed in nearly identical fashion. Average $\beta$ statistics were higher in P. zelicaon than in E. propertius, despite smaller sample sizes for $P$. zelicaon (owing to the relative difficulty of specimen collection). These comparative diversity results, both in terms of raw SNP counts per 1,000 bases and $\beta$, support previous findings that overall genetic polymorphism is higher for P. zelicaon than E. propertius [22].

As was found for Eucalyptus grandis, the $\beta$ distributions are all right-skewed (Table 4), suggesting purifying selection for the majority of genes [12]. $\beta_{t}$ is slightly negatively correlated with the number of species hit $(0,1,2$, or 3 of B. mori, H. erato, and D. melanogaster) for E. propertius, suggesting that lineage and species specific genes are more diverse for $E$. propertius $(r=-0.154, p<0.0001)$. A similar, but weaker and non-significant, trend is found for P. zelicaon $(r=-0.0254, p<0.054)$.

As has been noted before [38], different assembly programs can produce very different results, as seen in Table 1. While none of the assembly programs currently in widespread use are designed for ecoinformatics, Liang et al. have suggested that CAP3 is the best choice for ESTs [56]. However, Liang et al. did not consider the Celera Assembler, and our results suggest that new versions of the Celera Assembler may be more appropriate for data containing a diversity of genotypes.

For further comparison, we also assembled the E. propertius and P. zelicaon EST sets with the recently released Newbler assembler version 2.3 (Roche 454 Life Sciences), which has options specifically for transcriptome data. For E. propertius, Newbler produced 19,110 contigs of average length $637 \mathrm{bp}$ and 36,848 singletons with average (uncleaned) length $314 \mathrm{bp}$. For P. zelicaon, 25,336 contigs of average length $730 \mathrm{bp}$ and 20,926 singletons of average (uncleaned) length $297 \mathrm{bp}$ were produced. Newbler version 2.3 also produces a set of sequences known as "isotigs," arrangements of contigs meant to represent splice forms (similar to [55]). For E. propertius, 11,677 such isotigs with average length 1,238 bp were produced. 17,520 isotigs of average length $1,309 \mathrm{bp}$ were produced for P. zelicaon.

Another factor in successful transcriptome assembly is the sequencing technology used. In our application, the 454 Titanium chemistry sequencer produced average read lengths of about $400 \mathrm{bp}$. In contrast, the older 454 GS-20 platform used by Vera et al. produced reads averaging $110 \mathrm{bp}$ for the $M$. cinxia transcriptome [11]. To assess the effects of sequencing technology, we obtained M. cinxia ESTs from the Sequence Read Archive (SRA: SRR000670 and SRR000671) and cleaned and assembled them similarly to our datasets. (The original assembly by Vera et al. used SeqmanPro, a proprietary assembler from DNAStar.) After cleaning, 575,313 ESTs of average length $100 \mathrm{bp}$ remained. Our assembly produced 34,921 contigs (average length $141 \mathrm{bp}$ ), and 27,468 singletons (average length $81 \mathrm{bp}$ ). The fact that this assembly size is different from that produced by Vera et al. indicates that reanalysis of data may be important as new bioinformatics tools and assemblers become available.

Comparison between the above $M$. cinxia assembly and that for P. zelicaon or E. propertius is complicated by multiple factors. First, these are different species with different patterns of diversity and expression. Second, even though the number of cleaned ESTs is similar, the shorter read lengths for $M$. cinxia ESTs provide less total sequence data, affecting the number of contigs obtained. Nevertheless, the similar aspects of these datasets (including that they were all sourced from several individuals) does suggest that longer read lengths can improve assembly quality.

\section{Conclusion}

We reported larval transcriptome sequences and assemblies for butterflies of ecological importance: Erynnis propertius (Lepidoptera: Hesperiidae) and Papilio zelicaon (Lepidoptera: Papilionidae). As the immediate aim was construction of a microarray enabling comparison of transcribed genes under alternative climate treatments and of populations of differing geographic locations, steps were taken to maximize gene discovery within the larval stage.

Long read lengths produced by the 454 FLX Titanium sequencing platform and experimentation with assembly techniques produced high quality assemblies with few singletons. Over ten percent of putative B. mori orthologs in E. propertius and P. zelicaon cover at least $50 \%$ of the corresponding silkworm gene, as measured by ortholog hit ratio. Gene ontology annotation suggests that transcripts were broadly sampled, and comparisons with Bombyx mori and other related model species indicate that many genes were found--both species had hits to over $50 \%$ of the B. mori protein dataset.

Although the ortholog hit ratio does not consider the effects of alternative splicing (unless alternative splice forms also exist in the reference dataset), it appears to be an excellent method for the comparative assessment of assemblies. Using this measure, as well as simpler measures such as contig and singleton count, we found the Celera Assembler to be an effective tool for handling population-level datasets, particularly when custom parameters are used. 
454 sequencing and assembly has proven an effective platform for SNP discovery [3,11-14,41,57]. Variant regions detected with the Celera Assembler may prove useful for population-level studies, further supporting Celera Assembler for this type of data. Significantly, the discovery of $\sim 36 \mathrm{~K}$ high quality SNPs for E. propertius and $\sim 62 \mathrm{~K} \mathrm{SNPs} \mathrm{for} \mathrm{P.} \mathrm{zelicaon} \mathrm{will} \mathrm{facilitate} \mathrm{future} \mathrm{stud-}$ ies of population structure and genetic causes of functional differences already found between populations $[22,24,25]$.

\section{Methods}

\section{Rearing and RNA Isolation}

Eggs laid by adult E. propertius and P. zelicaon females were hatched under conditions characteristic of native habitats in a greenhouse and then moved to Conviron growth chambers at the University of Notre Dame. Multiple individuals of each larval instar were collected through the final instar before pupation [20]. Individuals of the $2^{\text {nd }}, 3^{\text {rd }}$, and $4^{\text {th }}$ instars (E. propertius) and $3^{\text {rd }}$ and $4^{\text {th }}$ instars (P. zelicaon) were exposed to a heat stress of 35 degrees for 60 minutes and a cold stress of 0 degrees for 120 minutes. Individuals in the $5^{\text {th }}$ and $6^{\text {th }}$ instar of $E$. propertius and $3^{\text {rd }}$ and $5^{\text {th }}$ instars of $P$. zelicaon were exposed to a desiccation agent (silica gel) for 120 minutes. In addition, some of the collected larvae of $P$. zelicaon were fed Petroselinum crispum and others were fed Lomatium utriculatum. The former contains higher concentrations of linear furanocoumarins, a defensive compound against herbivores, than the latter [58]. After treatment, larvae were frozen in liquid nitrogen and stored at $-80^{\circ} \mathrm{C}$.

Whole-body RNA from these frozen individuals was extracted using an RNA Easy kit (QIAGEN Inc.) over a period of two months. Prior to library construction, pooling was done by adjusting sample contributions to equimolar amounts of total RNA per individual.

\section{Library Construction and 454 Sequencing}

Library construction was performed by Express Genomics, Inc. (Frederick, Maryland, USA). Poly(A)+RNA from the E. propertius and P. zelicaon total RNAs was isolated by two rounds of oligo(dT) selection with oligo(dT) coated magnetic particles (Seradyn, Inc.).

From the poly $(\mathrm{A})+\mathrm{RNA}$ mRNA, cDNA libraries were constructed by using an oligo dT primer-adapter containing a Not I site and Moloney Murine Leukemia Virus Reverse Transcriptase (M-MLV RT) to prime and synthesize first strand cDNA. This process includes only one round of reverse transcription. After the second strand was synthesized, the double stranded cDNA was size fractionated $(>1.4 \mathrm{~kb})$ and cloned directionally into the Not I and Eco RV sites of the pExpress 1 vector. From one bulk ligation (300 ng of pExpress 1 vector, Not I-Eco RV cut, and 120 ng of Not I digested cDNA per $120 \mu \mathrm{l}$ of ligation), followed by electroporation into $\mathrm{T} 1$ phage resistant E. coli, primary clones were produced.

Normalized cDNA libraries were produced from the primary cDNA libraries. Biotinylated driver RNA produced from the T7 RNA polymerase promoter and single-stranded (ss) target DNA produced from the F1 ori were hybridized to each other at a low Cot (concentration of driver times the time of hybridization) value. The RNA:DNA hybrids were removed by phenol extraction and the remaining ss target DNA was converted to double-stranded DNA (dsDNA) with a repair oligo and Taq DNA polymerase. After electroporation of the dsDNA into $\mathrm{T} 1$ phage resistant $E$. coli, primary clones were produced.

The E. propertius and P. zelicaon normalized library DNAs were digested with Not I and in vitro RNA transcripts were produced using the SP6 RNA polymerase promoter. Then, first strand cDNA was made from these transcripts using a modified primer adapter that reduces the size of the poly A sequence (to about 20 As). After the second strand was synthesized, the double stranded (ds) cDNA was blunt ended and size fractionated. This ds cDNA was resuspended in TE, $\mathrm{pH} 8.0$, to between 110$125 \mathrm{ng} / \mathrm{ml}$.

The pooled sample for each species was run on onehalf of a plate on a 454 FLX Titanium machine at the Research Technology Support Facility at Michigan State University (East Lansing, Michigan, USA). EST sequences and quality scores are available from the National Center for Biotechnology Information (NCBI) Sequence Read Archive, accessions SRR035432.1 (E. propertius) and SRR035433.1 (P. zelicaon).

\section{Cleaning and Assembly}

ESTs were cleaned and vector trimmed with SeqClean [36] using both the NCBI Univec-Core database and the pExpress 1 vector (using the $-\mathrm{v}$ option to look for vector contamination), and the ESTs were scanned for E. coli (strain K-12, substrain MG1655) contamination (using the $-\mathrm{s}$ option for contamination screening). The default minimum read length cutoff of $100 \mathrm{bp}$ was used (using a larger, 200 bp cutoff did not drastically alter the singleton length distributions seen in Figures 1(a) and 1(b), data not shown).

Cleaned ESTs were assembled with CAP3 [37] and Celera Assembler [39,51], using two parameter sets each. Default CAP3 settings include -p 90 -h 20; the custom parameter settings used were $-\mathrm{p} \quad 85 \quad-\mathrm{h} \quad 90$. The CAP3 - p option specifies overlap percent identity cutoff, while the $-\mathrm{h}$ option specifies the maximum alignment overhang percentage. The recommended settings for Celera Assembler on 454 FLX Titanium chemistry are as follows: overlapper $=$ mer (use a seed and extend 
overlap algorithm), unitigger $=$ bog (use a best overlap graph approach for building unitigs), utgErrorRate $=0.03$ (the error rate above which the unitigger discards overlaps), dooverlapTrimming $=1$ (perform overlap based trimming) [40]. The final Celera assemblies used the following parameters: overlapper $=$ mer, unitigger $=$ bog, utgErrorRate $=0.07$, ovlerrorRate $=0.07$, cnsErrorRate $=0.07$, dooverlapTrimming $=1$. The parameter ovlErrorRate specifies an error rate above which the overlap module of the Celera Assembler will not report overlaps. Thus, the custom settings for both CAP3 and Celera Assembler allow for more tolerance of sequence divergence in assembling contigs.

\section{Gene Ontology Terms Distribution}

Gene ontology terms were assigned to unigenes and $B$. mori predicted proteins by via BLAST against the nonredundant nucleotide database NR (obtained September 20,2009 ) and analyzing the results using the Blast2GO tool [59]. These terms were mapped to high level gene ontology categories with CateGOrizer [60], using the "Aqua" categorization.

\section{SNP Detection}

Single nucleotide polymorphisms were identified by independently analyzing each column of the multiple alignments produced for contigs during the assembly process. Both the "loose" criterion, designed to maximize the discovery of rare alleles, and the "strict" criterion, designed to minimize the possibility of false positive identification, required that the consensus position not be a gap in the multiple alignment, and that there be at least two distinct nucleotide alleles present for that column.

The loose criterion required only that each of the two most common alleles be found in at least two ESTs. The strict criterion required that the minority allele (the second most common nucleic allele) be found in at least $25 \%$ of the ESTs covering that position, and that the total coverage at that position be at least $6 \times$. The loose SNP criterion is prescribed by Long et al. [61] as an effective method for SNP discovery in EST projects and is used as a secondary criterion by Barbazuk et al. [3]. The strict criterion is very similar to that used by Vera et al. [11]. Because it requires $25 \%$ minority allele coverage in highly covered areas, it is less sensitive to false positives than the loose criterion.

\section{Metatranscriptomic Contamination}

Unigenes not having hits to one of $D$. melanogaster, $B$. mori, H. erato, M. cinxia, or P. xuthus (see Annotation) were compared against the NCBI Non-Redundant protein set NR (obtained September 20, 2009) using BLASTX (cutoff 1e-5). Best hits were parsed using
MEGAN version 3.8 [62]. Assigned hits were counted at the kingdom taxonomic level, with the Min Support option set to 1 (such that every hit with bitscore greater than the default cutoff of 35.0 is assigned to a taxa).

\section{Clustering}

Unigenes were clustered based on BLAST similarity to other unigenes of the same species, $B$. mori, and $H$. erato protein databases. Each unigene, B. mori protein, and $H$. erato protein was considered as a vertex in a graph (representing sequence similarity between elements of the datasets). Each unigene was connected to its best BLASTN unigene match, best BLASTX B. mori hit, and best BLASTX $H$. erato hit ( $e<1 \mathrm{e}-5$ cutoff) by an undirected edge in the graph. Clusters of unigenes were those present in the same connected component of the graph-that is, unigenes that are reachable from each other by following a path in the graph [63]. For example, if unigene $A$ had a hit to unigene $B$, and unigenes $B$ and $C$ both had hits to a $B$. mori protein $\mathrm{X}$, then $\mathrm{A}, \mathrm{B}$, and $\mathrm{C}$ would be considered a cluster.

\section{Authors' contributions}

STO performed most of the bioinformatics analysis and drafted the manuscript. JDK supervised fieldwork, specimen collection, and cDNA sequencing. RDC helped assemble and analyze the data. NFL participated in design, data analysis, assembly validation, and drafting the manuscript. SJE helped conceive the study, coordinated analysis, and helped draft the manuscript. JJH conceived the study and helped coordinate and draft the manuscript. All authors read and approved the final manuscript.

\section{Acknowledgements}

This work was supported by the Office of Science (BER), US Department of Energy, Grant no. DE-FG02-05ER to JJH, and the Arthur J. Schmitt Foundation. We also thank Katrina Hill, Jessica Keppel, Chris Lambert, Shannon Pelini, Aubrey Podell, Sean Ryan, and Megan Stachura for field and laboratory assistance. Finally, we thank three anonymous reviewers for insightful comments.

\section{Author Details}

'Department of Computer Science and Engineering, University of Notre Dame, Notre Dame, IN, USA and 2Department of Biological Sciences, University of Notre Dame, Notre Dame, IN, USA

Received: 24 August 2009 Accepted: 17 May 2010

Published: 17 May 2010

\section{References}

1. Adams M, Kelley J, Gocayne J, Dubnick M, Polymeropoulos M, Xiao H, Merril C, Wu A, Olde B, Moreno R, Kerlavage A, McCombie W, Venter J: Complementary DNA sequencing: Expressed sequence tags and the human genome project. Science 1991, 252:1651-1656.

2. Rudd S: Expressed sequence tags: alternative or complement to whole genome sequences? Trends in Plant Science 2003, 8(7):321-329.

3. Barbazuk B, Emrich S, Chen H, Li L, Schnable P: SNP discovery via 454 transcriptome sequencing. The Plant Journal 2007, 51(5):910-918.

4. Emrich S, Barbazuk W, Li L, Schnable P: Gene discovery and annotation using LCM-454 transcriptome sequencing. Genome Res 2007, 17:69-73.

5. Mao C, Evans $C$, Jensen R, Sobral B: Identification of new genes in Sinorhizobium meliloti using the Genome Sequencer FLX system. BMC Microbiology 2008, 8:72+.

6. Lee A, Hansen KD, Bullard J, Dudoit S, Sherlock G: Novel Low Abundance and Transient RNAs in Yeast Revealed by Tiling Microarrays and Ultra High-Throughput Sequencing Are Not Conserved Across Closely Related Yeast Species. PLoS Genet 2008, 4(12):e1000299+. 
7. Khajuria C, Zhu Y, Chen M, Buschman L, Higgins R, Yao J, Creso A, Siegfried B, Muthukrishnan S, Zhu K: Expressed sequence tags from larval gut of the European corn borer (Ostrinia nubilalis): Exploring candidate genes potentially involved in Bacillus thuringiensis toxicity and resistance. BMC Genomics 2009, 10:286+.

8. Ohtsu K, Smith M, Emrich S, Borsuk L, Zhou R, Chen T, Zhang X, Timmermans M, Beck J, Buckner B, Janick-Buckner D, Nettleton D, Scanlon M, Schnable P: Global gene expression analysis of the shoot apical meristem of maize (Zea mays L.). The Plant Journal 2007, 52(3):391-404.

9. Torres T, Metta M, Ottenwälder BCS: Gene expression profiling by massively parallel sequencing. Genome Research 2008, 18:172-177.

10. Hornshøj H, Bendixen E, Conley L, Andersen P, Hedegaard J, Panitz F, Bendixen C: Transcriptomic and proteomic profiling of two porcine tissues using high-throughput technologies. BMC Genomics 2009, 10:30.

11. Vera CJ, Wheat C, Fescemyer H, Frilander M, Crawford D, Hanski I, Marden $\mathrm{J}$ : Rapid transcriptome characterization for a nonmodel organism using 454 pyrosequencing. Molecular Ecology 2008, 17(7):1636-1647.

12. Novaes E, Drost D, Farmerie W, Pappas G, Grattapaglia D, Sederoff R, Kirst M: High-throughput gene and SNP discovery in Eucalyptus grandis, an uncharacterized genome. BMC Genomics 2008, 9:

13. Cheung F, Win J, Lang J, Hamilton J, Vuong H, Leach J, Kamoun S, André Lévesque C, Tisserat N, Buell C: Analysis of the Pythium ultimum transcriptome using Sanger and Pyrosequencing approaches. $B M C$ Genomics 2008, 9:542+.

14. Meyer E, Aglyamova G, Wang S, Carter J, Abrego D, Colbourne J, Willis B, Matz M: Sequencing and de novo analysis of a coral larval transcriptome using 454 GS-FLX. BMC Genomics 2009, 10:219+.

15. Roeding F, Borner J, Kube M, Klages S, Reinhardt R, Burmester T: A 454 sequencing approach for large scale phylogenomic analysis of the common emperor scorpion (Pandinus imperator). Mol Phylogenet Evol 2009, 53(3):826-834.

16. Weber A, Weber K, Carr K, Wilkerson C, Ohlrogge J: Sampling the Arabidopsis transcriptome with massively parallel pyrosequencing. Plant Physiol 2007, 144:32-42.

17. Papanicolaou A, Joron M, McMillan W, Blaxter M, Jiggins C: Genomic tools and CDNA derived markers for butterflies. Molecular Ecology 2005 14(9):2883-2897.

18. Ozaki K, Utoguchi A, Yamada A, Yoshikawa H: Identification and genomic structure of chemosensory proteins (CSP) and odorant binding proteins (OBP) genes expressed in foreleg tarsi of the swallowtail butterfly Papilio xuthus. Insect Biochem Mol Bio/ 2008, 38(11):969-976.

19. Guppy C, Shepard R: Butterflies of British Columbia Vancouver: UBC Press; 2001

20. Prior K, Hellmann J: The ecology and life history of Erynnis Propertius, a threatened oak feeding butterfly. Canadian Entomology 2009, 141:161-171.

21. Scott J: The Butterflies of North America: a Natural History and Field Guide Stanford, California: Stanford University Press; 1992

22. Zakharov E, Hellmann J: Genetic differentiation across a latitudinal gradient in two co-occurring butterfly species: revealing population differences in a context of climate change. Molecular Ecology 2008, 17:189-208

23. Zakharov E, Lobo N, Nowak C, Hellmann J: Introgression as a likely cause of mtDNA paraphyly in two allopatric skippers (Lepidoptera: Hesperiidae). Heredity 2009, 102:590-599.

24. Hellmann J, Pelini S, Prior K, Dzurisin J: The response of two butterfly species to climatic variation at the edge of their range and the implications for poleward range shifts. Oecologia 2008, 157(4):583-592.

25. Pelini S, Dzurisin J, Prior K, Williams C, Marsicos T, Sinclair B, Hellmann J: Translocation experiments with butterflies reveal limits to enhancement of poleward populations under climate change. Proceedings of the National Academy of Sciences 2009.

26. Zakharov E, Hellmann J: Characterization of 17 polymorphic microsatellite loci in the Anise swallowtail, Papilio zelicaon (Lepidoptera: Papilionidae), and their amplification in related species. Molecular Ecology Notes 2007, 7:144-146.

27. Zakharov E, Hellmann J, Romero-Severson J: Microsatellite loci in the Propertius duskywing, Erynnis propertius (Lepidoptera: Hesperiidae), and related species. Molecular Ecology Notes 2007, 7(2):266-268.
28. Li W, Berenbaum M, Schuler M: Molecular analysis of multiple CYP6B genes from polyphagous Papilio species. Insect Biochemistry and Molecular Biology 2001, 31(10):999-1011.

29. Li W, Petersen R, Schuler M, Berenbaum M: CYP6B cytochrome P450 monooxygenases from Papilio canadensis and Papilio glaucus: potential contributions of sequence divergence to host plant associations. Insect Molecular Biology 2002, 11(6):543-551.

30. Boggs C, Watt W, Ehrlich P: Butterflies: ecology and evolution taking flight Chicago, IL: University of Chicago Press; 2003

31. Boggs C: Reproductive strategies of female butterflies: variation in and constraints on fecundity. Ecological Entomology 1986, 11:7-15.

32. Leather S: Size, reproductive potential and fecundity in insects: Things aren't as simple as they seem. Oikos 1988, 51:386-389.

33. Stockhoff B: Starvation resistance of gypsy moth, Lymantria dispar (L.) (Lepidoptera: Lymantriidae): tradeoffs among growth, body size, and survival. Oecologia 1991, 88(3):422-429.

34. Oberhauser K: Fecundity, lifespan and egg mass in butterflies: Effects of male-derived nutrients and female size. Func Ecology 1997, 11:166-175.

35. Hahn D, Denlinger D: Meeting the energetic demands of insect diapause: Nutrient storage and utilization. Journal of Insect Physiology 2007, 53(8):760-773

36. DFCl Gene Indices Software Tools [http://compbio.dfci.harvard.edu/tgi/ software/

37. Huang X, Madan A: CAP3: A DNA sequence assembly program. Genome Research 1999, 9:868-877.

38. Bouck A, Vision T: The molecular ecologist's guide to expressed sequence tags. Molecular Ecology 2007, 16:907-924.

39. Myers E, Sutton G, Delcher A, Dew I, Fasulo D, Flanigan M, Kravitz S, Mobarry C, Reinert K, Remington K, Anson E, Bolanos R, Chou H, Jordan C, Halpern A, Lonardi S, Beasley E, Brandon R, Chen L, Dunn Z, PJ Lai, Liang Y, Nusskern D, Zhan M, Zhang Q, Zheng X, Rubin G, Adams M, Venter J: A whole-genome assembly of Drosophila. Science 2000, 287(5461):2196-2204

40. Celera Assembler SFF Standard Operating Procedures [http:// sourceforge.net/apps/mediawiki/wgs-assembler/ index.php?title=SFF SOP

41. Hale M, McCormick C, Jackson J, DeWoody J: Next-generation pyrosequencing of gonad transcriptomes in the polyploid lake sturgeon (Acipenser fulvescens): the relative merits of normalization and rarefaction in gene discovery. BMC Genomics 2009, 10:203.

42. Duan J, Li R, Cheng D, Fan W, Zha X, Cheng T, Wu Y, Wang J, Mita K, Xiang Z, Xia Q: SilkDB v2.0: a platform for silkworm (Bombyx mori) genome biology. Nucl Acids Res 2009:gkp801+

43. Altschul S, Madden T, Schaffer A, Zhang J, Zhang Z, Miller W, Lipman D: Gapped BLAST and PSI-BLAST: a new generation of protein database search programs. Nucleic Acid Res 1997, 25:3389-3402

44. Lottaz C, Iseli C, Jongeneel C, Bucher P: Modeling sequencing errors by combining Hidden Markov models. Bioinformatics 2003, 16:ii103-ii1 12

45. Tweedie S, Ashburner M, Falls K, Leyland P, McQuilton P, Marygold S, Millburn G, Osumi-Sutherland D, Schroeder A, Seal R, Zhang H, The FlyBase Consortium: FlyBase: enhancing Drosophila Gene Ontology annotations. Nucleic Acids Research 2009, 37:D555-D559.

46. Papanicolaou A, Gebauer-Jung S, Blaxter M, Owen McMillan W, Jiggins C ButterflyBase: a platform for lepidopteran genomes. Nucleic Acid Research 2008, 36:D582-D587.

47. Jiggins C: personal communication 2009.

48. Wiegmann B, Trautwein M, Kim J, Cassel B, Bertone M, Winterton S, Yeates $D$ : Single-copy nuclear genes resolve the phylogeny of the holometabolous insects. BMC Biology 2009, 7(34):34+.

49. Cheng T, Xia Q, Qian J, Liu C, Lin Y, Zha X, Xiang Z: Mining single nucleotide polymorphisms from EST data of silkworm, Bombyx mori, inbred strain Dazao. Insect Biochemistry and Molecular Biology 2004 34(6):523-530

50. Berger J, Suzuki T, Senti KA, Stubbs J, Schaffner G, Dickson BJ: Genetic mapping with SNP markers in Drosophila. Nature Genetics 2001, 29(4):475-481.

51. Denisov G, Walenz B, Halpern AL, Miller J, Axelrod N, Levy S, Sutton G: Consensus generation and variant detection by Celera Assembler. Bioinformatics (Oxford, England) 2008, 24(8):1035-1040. 
52. Watanabe M: Multiple matings increase the fecundity of the yellow swallowtail butterfly, Papilio xuthus L., in summer generations. Journal of Insect Behavior 1988, 1:17-27.

53. Sims S: Aspects of mating frequency and reproductive maturity in Papilio zelicaon. American Midland Naturalist 1979, 102:36-50.

54. Watterson G: On the number of segregating sites in genetical models without recombination. Theoretical Population Biology 1975, 7(2):256-276.

55. Heber S, Alekseyev M, Sze S, Tang H, Pevzer P: Splicing graphs and the EST assembly problem. Bioinformatics 2002, 18:S181-S188.

56. Liang F, Holt I, Pertea G, Karamycheva S, Salzberg S, Quackenbush J: An optimized protocol for analysis of EST sequences. Nucl. Acids Res 2000, 28(18):3657-3665.

57. Bainbridge M, Warren R, Hirst M, Romanuik T, Zeng T, Go A, Delaney A Griffith M, Hickenbotham M, Magarini V, Mardis E, Sadar M, Siddiqui A, Marra M, Jones S: Analysis of the prostate cancer cell line LNCaP transcriptome using a sequencing-by-synthesis approach. $B M C$ Genomics 2006, 7:246+.

58. Berenbaum M: Coumarins and Caterpillars: A Case for Coevolution. Evolution 1983, 37:163-179.

59. Götz S, García-Gómez J, Terol J, Williams T, Nagaraj S, Nueda M, Robles M, Talón M, Dopazo J, Conesa A: High-throughput functional annotation and data mining with the Blast2GO suite. Nucleic acids research 2008, 36(10):3420-3435.

60. Hu Z, Jie B, Reecy J: CateGOrizer: A Web-Based Program to Batch Analyze Gene Ontology Classification Categories. Online Journal of Bioinformatics 2008, 9(2):

61. Long A, Beldade P, Macdonald S: Estimation of population heterozygosity and library construction-induced mutation rate from expresed sequence tag collections. Genetics 2007, 176:711-714.

62. Huson D, Auch A, Qi J, Schuster S: MEGAN analysis of metagenome data. Genome Research 2007, 17:377-386.

63. Cormen T, Leiserson C, Rivest R, Stein C: Introduction to Algorithms 2nd edition. MIT Press, McGraw-Hill Book Company; 2000.

Cite this article as: O'Neil et al., Population-level transcriptome sequencing of nonmodel organisms Erynnis propertius and Papilio zelicaon BMC Genomics 2010, 11:310

Submit your next manuscript to BioMed Centra and take full advantage of:

- Convenient online submission

- Thorough peer review

- No space constraints or color figure charges

- Immediate publication on acceptance

- Inclusion in PubMed, CAS, Scopus and Google Scholar

- Research which is freely available for redistribution

Submit your manuscript at www.biomedcentral.com/submit
C) BioMed Central 